\title{
Studies on Structural and Magnetic Properties in Co/Sm Multilayers
}

\author{
Erwin \\ Department of Physics Faculty of Mathematics and Natural Sciences Riau University, \\ Bina Widya Campus, Simpang Panam Km. 12.5, Pekanbaru 28293 \\ Diterima 12-12-2009 \\ Disetujui 08-05-2010
}

\begin{abstract}
$\mathrm{Co} / \mathrm{Sm}$ multilayer films with structure of $20[\mathrm{Co}(\mathrm{x} \mathrm{nm}) / \mathrm{Sm}(1.2 \mathrm{~nm})]$ where $\mathrm{x}=1.1,2.2$ and $4.2 \mathrm{~nm}$ and $20[\mathrm{Co}$ (4.2 $\mathrm{nm}) / \mathrm{Sm}(x \mathrm{~nm})]$ where $x=1.2 \mathrm{~nm}$ to $7.5 \mathrm{~nm}$ were fabricated using dc magnetron sputtering. Each multilayer film consisted of 20 bilayers of Co layers with various thicknesses sandwiched with Sm layers. The application of low angle X-ray diffraction measurements to the characterization of these multilayers is described. The periodic layered structure with sharp interfaces was observed for all multilayer films. The measured magnetization values are lower than the values calculated in terms of the nominal concentration of cobalt in the multilayers. This implies significant "mixing" at small film thickness. The formation of a high magneto crystalline anisotropy of CoSm alloy at the interfaces, as a result of interdiffusion between $\mathrm{Co}$ and $\mathrm{Sm}$ layers was considered to be responsible for the increase of the coercivity for $\mathrm{Co} / \mathrm{Sm}$ multilayer.
\end{abstract}

Keywords: coercivity, magnetic, microstructure, multilayer films, structure

\section{INTRODUCTION}

In recent year, cobalt based alloys in the form of thin films and thin film multilayer structures consist of alternate magnetic and non magnetic layers of manometer thickness have attracted much attention due to their magnetic properties. One important application of such films is as high-density magnetic recording media. Many such alloys are based on the CoCr binary system with often multinary additions of such elements as $\mathrm{Pt}$, Ta and $\mathrm{B}$. An alternative binary system is that of CoRe alloys (Re-rare earth), and in particular the CoSm system. This allows, in principle and in equilibrium, the formation of a series of CoSm compounds with properties that reflect those of the compounds formed in bulk CoSm permanent magnets namely $\mathrm{Co}_{17} \mathrm{Sm}_{2}, \mathrm{Co}_{5} \mathrm{Sm}$ and $\mathrm{Co}_{7} \mathrm{Sm}_{2}$. There is also the additional interest in this system in that CoSm alloys can be deposited in the amorphous phase at Sm above about 20 atomic \%.

According to (Murdock et al., 1992), magnetic recording media for high-density magnetic data storage with low noise require a material consisting of small and magnetically isolated grains. In small grain sizes of about $10 \mathrm{~nm}$ or below, high magneto crystalline

Telp: +6281371302206

Email: erwin_amirruddin@yahoo.com anisotropy is needed in order to avoid thermal fluctuation that tends to destabilize the magnetization of the recorded bits (1997).

Early attempts to grow CoSm alloys in the form of thin films exhibiting large coercivity values were carried out by (Theuerer et al., 1969; Gronou et al., 1983), reported that CoSm films with high coercivity had been prepared by flash evaporation on glass substrates. They suggested that these films could be used as a highdensity magnetic recording medium. Some recording experiments have been investigated, for example, by (Velu \& Lambeth,1992; Velu et al., 1994), However, the growth characteristics of CoSm alloys including the concentration range (1982), the epitaxial relation between CoSm alloy films and underlayer materials,(1998; 1991), interaction effects (1998; 2000), and the magnetic switching volume (1995), suggest that improved magnetic properties of CoSm films could be obtained by utilization and optimisation of appropriate deposition conditions.

Several methods have been used in growing CoSm thin films. They include sputtering, evaporation and plasma spraying. Thin films with controlled geometry and surface smoothness as well as composition can be easily fabricated by the sputtering method (1991). It is commonly observed that sputter deposition of CoSm 
thin films below $600^{\circ} \mathrm{C}$ usually produces alloys with an amorphous structure (1987). The amorphous structure of these alloys may limit their anisotropy and thus their coercivity. A higher value of coercivity can be simply achieved by introducing the crystalline state in the CoSm alloy by deposition with elevated substrate temperature or by post annealing of as-deposited films.

With regard to multilayer films, which are composed usually of ferromagnetic and non-magnetic thin layers, the interfacial mixing may exhibit novel magnetic properties. $(1989$; 1990) Work on multilayer films in which the nonmagnetic layer is composed of a rare earth and metalloid elements has been carried out for ferromagnetic materials forming the ferromagnetic layers. It was reported by Petford-Long et al., (1990) that interfacial mixing exists for the Co/W structure that affects the magnetic properties.

This paper is concerned with the investigation of magnetic and structural properties of $\mathrm{Co} / \mathrm{Sm}$ multilayer films. This study is driven by fundamental interest and by the need to design materials with magnetic properties which might be suited for high density magnetic recording media. Multilayer films of alternatively deposited ferromagnetic and nonmagnetic layers are also proving to be important for understanding the fundamental physics of interacting magnetic films.

\section{MATERIALS AND METHODS}

The Co/Sm multilayer films were deposited onto $\mathrm{Si}(100)$ substrates by dc magnetron sputtering with a sputtering pressure of $4 \times 10^{-8} \mathrm{mbar}$ of argon gas and the deposition rates were about $0.21 \mathrm{~nm} / \mathrm{s}$ and $0.31 \mathrm{~nm} / \mathrm{s}$ for Co and Sm respectively. The samples were divided into two groups. The first group of samples takes the form of 20[Co $(x \mathrm{~nm}) / \mathrm{Sm}(1.2 \mathrm{~nm})]$. Each multilayer film consisted of 20 bilayers of Co layers with various thicknesses sandwiched with $\mathrm{Sm}$ layers of $1.2 \mathrm{~nm}$ thick. The second group of multilayer films has the form of $20[\mathrm{Co}(4.2 \mathrm{~nm}) / \mathrm{Sm}(\mathrm{x} \mathrm{nm})]$ in which the samples have the same Co layer thickness of $4.2 \mathrm{~nm}$ and the Sm layer thickness is progressively increased from $1.2 \mathrm{~nm}$ to $7.5 \mathrm{~nm}$. Again, each multilayer film consists of 20 bilayers of $4.2 \mathrm{~nm}$ of Co layers sandwiched with $\mathrm{Sm}$ of various thicknesses.

The periodic structure i.e., sharp interfaces, in the samples of the samples was studied by small angle $X$ ray diffraction. The data obtained were processed with special computer program which is called XREALM software (1988). Layer thicknesses were determined by sputtering rate and from the low angle X-ray diffraction patterns. The magnetic properties of the samples were studied by alternating gradient force magnetometer (AGFM) and vibration sample magnetometer (VSM). High-resolution transmission electron microscopy (HRTEM) has been used to analyse the microstructure of some of the as-deposited samples.

\section{RESULT AND DISCUSSION}

Low Angle X-Ray Diffraction Profiles. The low angle $x$-ray diffraction technique was used to investigate the structure of $\mathrm{Co} / \mathrm{Sm}$ multilayer films. Figure 1 shows the low angle diffraction patterns for multilayer films with structure of $20[\mathrm{Co}(\mathrm{x} \mathrm{nm}) / \mathrm{Sm}(1.2 \mathrm{~nm})]$ where $\mathrm{x}=$ 1.1, 2.2, and 4.2. Computer simulations of the data, perform using XREALM softwar (1988) for multilayer films are included for comparison. In the simulated reflectivity profile, variables such as the layer thickness of the Co and Sm films, the roughness of the substrate and the layers, and the relative densities of substrate and films, can be adjusted so that one can obtain the closest fit to the experimental data. It can be seen that almost all the reflection peak positions of the multilayer films correspond to those in the simulated reflectivity profile. From the simulated reflectivity profile analysis, for example, the multilayer film with $x=1.1 \mathrm{~nm}$, it was found that the thickness of periodic bilayer was 2.22 $\mathrm{nm}$, which corresponds to the thickness of Co and Sm layers of $1.10 \mathrm{~nm}$ and $1.12 \mathrm{~nm}$ respectively.

From Figure 1, the multilayer films show several orders of diffraction peaks, indicating the periodic nature of the sample. For the multilayer film with $x=1.1 \mathrm{~nm}$, finite size oscillations (Kiessig fringes) persist to 5.5 degrees, implying that the overall surface of the multilayer film is smooth. However, for $x=2.2$, and 4.2 $\mathrm{nm}$ finite size oscillations are not very clear, suggesting that the surface of the films is not smooth. With increasing Co layer thickness, diffraction peaks shift to a smaller value of angle of incidence suggesting that the bilayer thickness is increased. From the position of low angle XRD peaks shown in Figure 1, the thickness of the periodic layer (bilayer) for all samples could be calculated. Table 1 shows the bilayer thickness for nominal, simulated and calculated (low angle XRD).

Magnetic Properties Figure 2 shows hysteresis loops measured at room temperature for multilayer films 


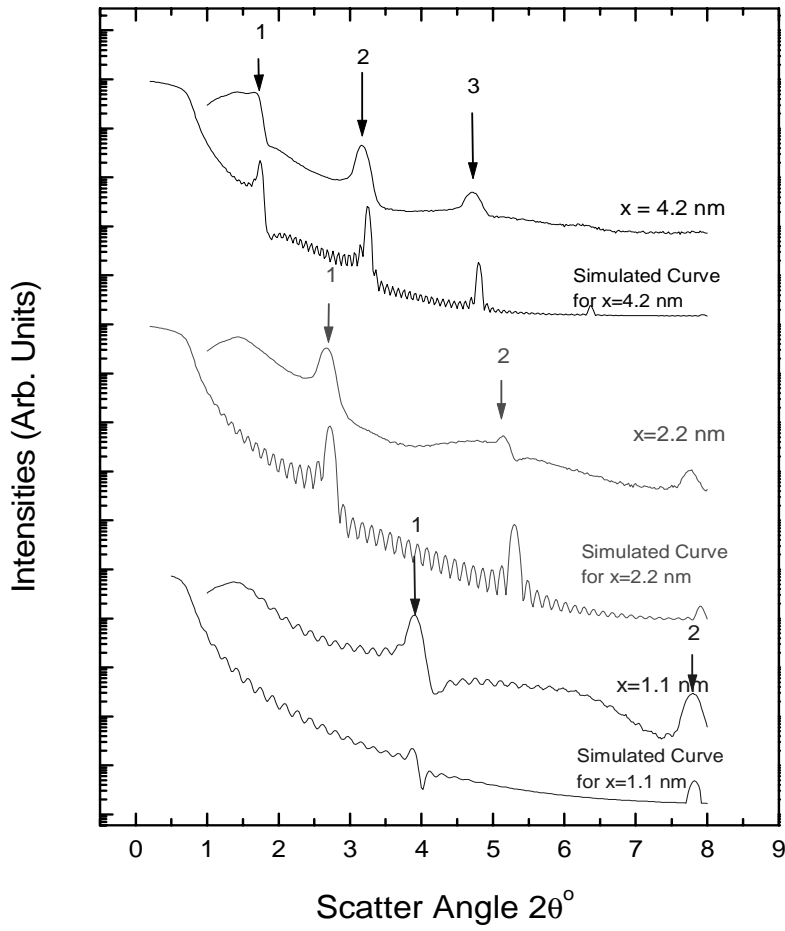

Figure 1. Low angle $x$-ray reflectivity profiles of multilayer films with structure of $20[\mathrm{Co}(\mathrm{x} \mathrm{nm}) / \mathrm{Sm}(1.2 \mathrm{~nm})]$. The order of the Bragg peaks is marked on some curves.

Table 1. Bilayer thickness of Co/Sm multilayer films with structure of 20[Co $(x \mathrm{~nm}) / \mathrm{Sm}(1.2 \mathrm{~nm})]$.

\begin{tabular}{cccc}
\hline \multirow{2}{*}{ Sample Code } & \multicolumn{3}{c}{ Bilayer Thickness ( nm ) } \\
\cline { 2 - 4 } & Nominal & $\begin{array}{c}\text { Simulated } \\
\text { Results }\end{array}$ & $\begin{array}{c}\text { Low Angle XRD } \\
\text { Results }\end{array}$ \\
\hline ML10A & 2.3 & 2.2 & $2.20 \pm 0.01$ \\
ML10B & 3.3 & 3.3 & $3.30 \pm 0.16$ \\
ML10C & 4.2 & 4.2 & $4.20 \pm 0.23$ \\
ML10D & 5.4 & 5.4 & $5.40 \pm 0.47$ \\
\hline
\end{tabular}

with the structure of 20[Co $(x \mathrm{~nm}) / \mathrm{Sm}(1.2 \mathrm{~nm})]$ for $\mathrm{x}=1.1,2.2$ and $4.2 \mathrm{~nm}$. For the multilayer film with Co layer thickness of $1.1 \mathrm{~nm}$, the coercivity is about 740 Oe. The hysteresis loop which has low remanence magnetization ratio (around 0.6) exhibits a small kink in booth field directions i.e., at near zero field indicating the presence of either a second magnetically soft phase or low field nucleation phenomena that initiate magnetization reversal (1997). The large value of coercivity of this multilayer film compared to those with thicker Co layers is interpreted as being due to possible interlayer mixing at a composition of about 3:1 (equivalent to the $\mathrm{Co}_{3} \mathrm{Sm}$ phase). The saturation magnetization value for this multilayer film is about 150 $\mathrm{emu} / \mathrm{cm}^{3}$, which is similar to that for the $\mathrm{Co}_{3} \mathrm{Sm}$ compound, hence the choice of $\mathrm{Co}_{3} \mathrm{Sm}$ as the 'mixing' composition.

The effect of a greater Co layer thickness on the loop for Co layer thickness of $2.2 \mathrm{~nm}$ is shown in Figure $2 \mathrm{~b}$. The coercivity of this multilayer decreases
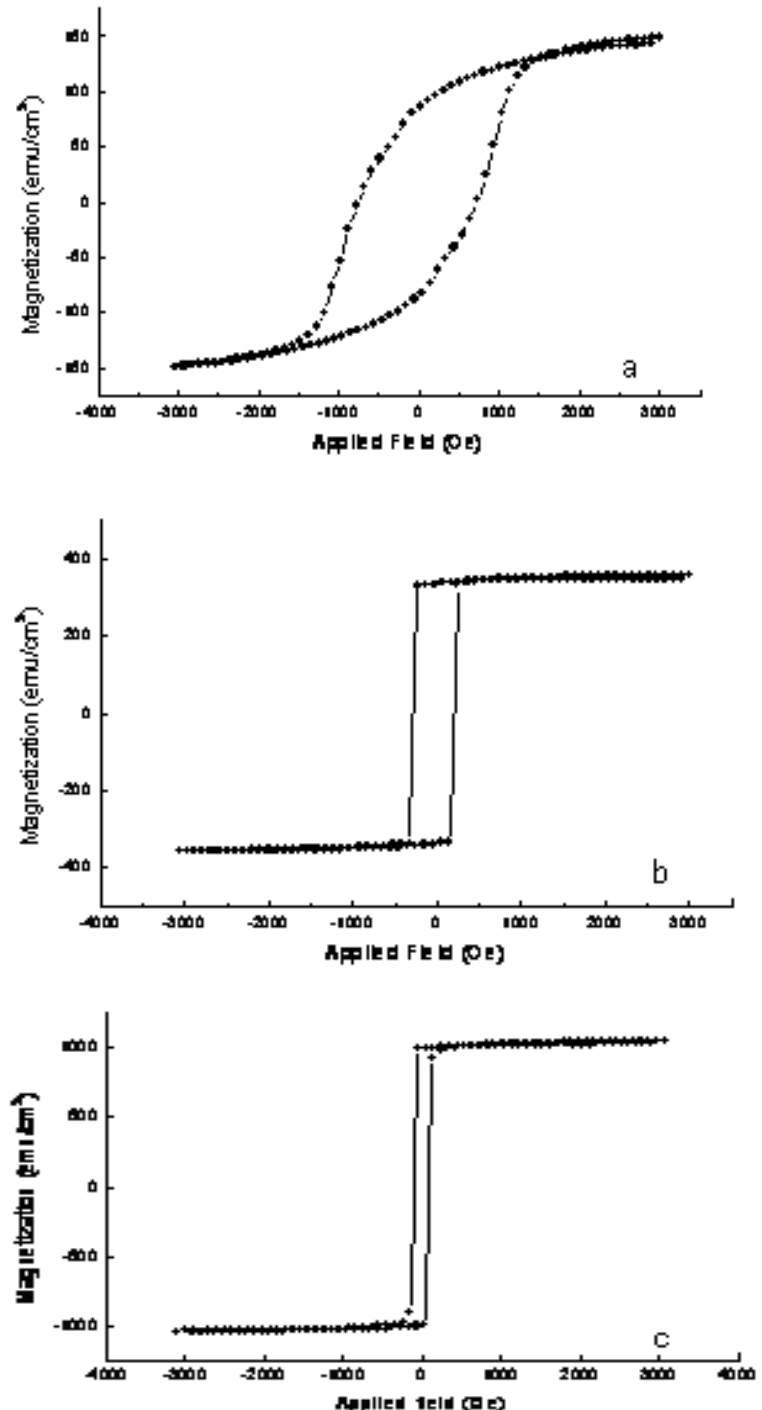

Figure 2 . Hysteresis toop measurea at room temperature tor multilayer films with structure of $20[\mathrm{Co}(x \mathrm{~nm}) / \mathrm{Sm}(1.2$ $\mathrm{nm}$ )], where (a) $\mathrm{x}=1.1 \mathrm{~nm}$, (b) $\mathrm{x}=2.2 \mathrm{~nm}$, and (c) $\mathrm{x}=$ $4.2 \mathrm{~nm}$.

very significantly to 230 Oe with a higher loop squareness (0.95). The saturation magnetization value for this multilayer film is about $350 \mathrm{emu} / \mathrm{cm}^{3}$. Increasing the Co layer thickness further $(4.2 \mathrm{~nm})$ leads to a further decrease in coercivity $(90 \mathrm{Oe})$. The magnetization value increased to about $1020 \mathrm{emu} / \mathrm{cm}^{3}$. The hysteresis loop exhibits a higher squareness (0.97) as shown in Figure 2c. An increase of magnetization value with increasing Co layer thickness is reasonable, and is due to an increase in the amount of the magnetic Co in the multilayer. However, the measured magnetization values are lower than the values calculated in terms of the nominal concentration of cobalt in the multilayers. This is particularly so for the smaller values of $x$, i.e., for $x=1.1 \mathrm{~nm}$. The calculated magnetization of about 670 $\mathrm{emu} / \mathrm{cm}^{3}$ compares to the measured value of $150 \mathrm{emu} /$ $\mathrm{cm}^{3}$. The respective values for $\mathrm{x}=2.2 \mathrm{~nm}$ are $900 \mathrm{emu} /$ 
$\mathrm{cm}^{3}$ and $350 \mathrm{emu} / \mathrm{cm}^{3}$ and for $\mathrm{x}=4.2 \mathrm{~nm}$ are $1090 \mathrm{emu} /$ $\mathrm{cm}^{3}$ and $1020 \mathrm{emu} / \mathrm{cm}^{3}$. This implies significant mixing at small film thickness.

The interaction effect given by $\delta M$ for the three multilayers is shown in Figure 3. For the multilayer film with $\mathrm{x}=1.1 \mathrm{~nm}$ the $\delta M$ shows a broad positive and then negative peak. This could be due to exchange coupling over an extended field range between the Co layers, but there is also a strong dipolar coupling which becomes obvious at larger fields and moderates the exchange coupling because the Co layers are close enough for significant magnetostatic dipolar coupling.
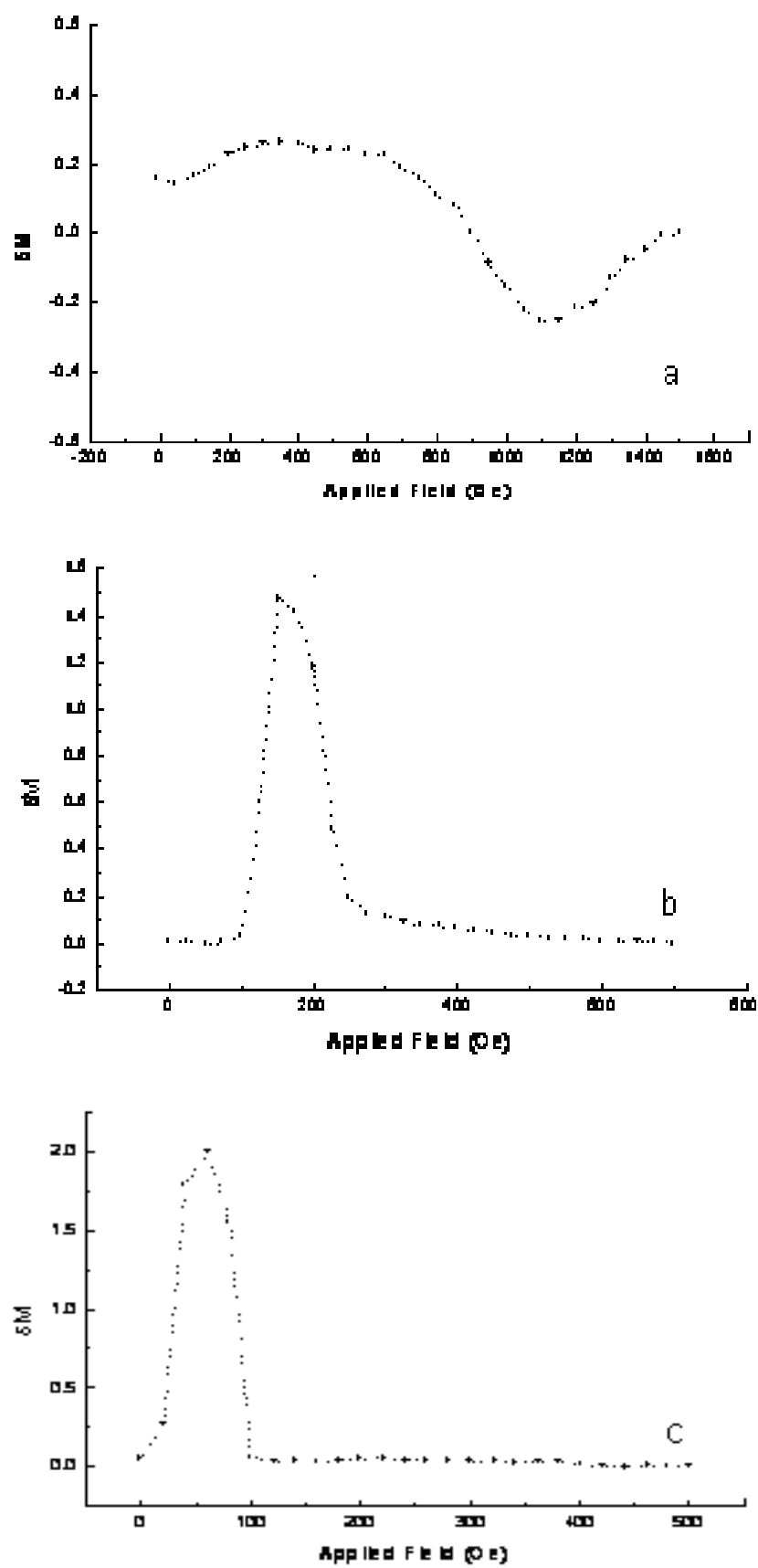

Figure 3. $\delta M$ curves as a function of applied field for multilayer

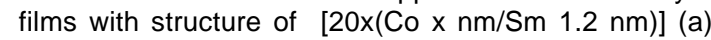
$x=1.1 \mathrm{~nm}$ (b) $x=2.2 \mathrm{~nm}$ and (c) $x=4.2 \mathrm{~nm}$.
The boardening of the positive $\delta M$ is due to the interlayer dipolar coupling in the multilayer film, which prevents any sharp cooperative reversal (2001). This result is consistent with the lower loop squareness, Figure 2(a).

For multilayer films with $\mathrm{x}=2.2$ and $4.2 \mathrm{~nm}$ the interaction effect $(\delta M)$ shows a very sharp positive peak. The narrow positive peak of $\delta M$ is indicative of stronger exchange coupling in the Co layers and possibly between the Co layers. This strong coupling dominates any dipolar coupling that is present. The exchange coupling is even stronger for the thickest Co layers, as shown in Figure 3(c). This result is consistent with higher loop squarenesses, Figure 2(b) and (c). It is difficult to assess the effect of interface roughness at the larger values of $x$ on the magnetic interactions in the films but any correlated or uncorrelated roughness would have some effect through the Néel-type mechanism/orange PEEL COUPLING (1962).

Microstructural Properties. A high-resolution transmission electron microscopy (HRTEM) crosssectional image of a multilayer film with structure of $20[\mathrm{Co}(1.1 \mathrm{~nm}) / \mathrm{Sm}(1.2 \mathrm{~nm})]$ is shown in Figure 4. The sharp periodic layers of $\mathrm{Co}$ and $\mathrm{Sm}$ can be clearly distinguished as a result of the difference in electron scattering of the sample. The darker Co layers are clearly separated from the brighter Sm layers. A regular periodicity without discontinuity of the bilayers structure can be clearly seen, especially close to the substrate. Another feature that can be noticed from Figure 4 is that the roughness of the layers increases with distance from the substrate. The bilayer thickness derived from the cross-sectional HRTEM images of the multilayer films is in good agreement with the one obtained from the low angle $X$-ray diffraction measurement shown in Figure 1. The thicknesses of the Co and Sm layers

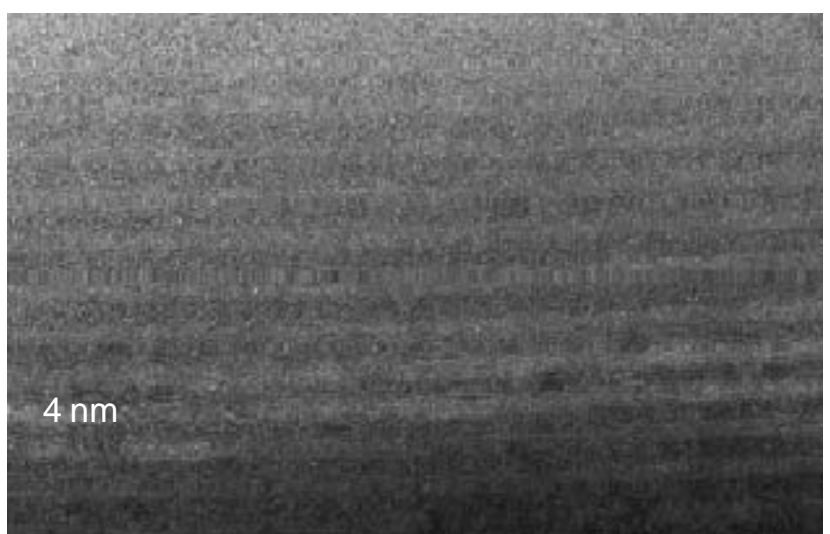

Figure 4. High resolution transmission electron microscopy (HRTEM) cross sectional image of multilayer films with structure of $20\left[C_{0}(1.1 \mathrm{~nm}) / \mathrm{Sm} 1.2 \mathrm{~nm}\right)$ 
given by Figure 4 are also well agree with the nominal deposition values.

Figure 5 shows a highly magnified micrograph of this multilayer film. Within the Co layers some crystalline grains are observed. These crystalline grains are a few nanometres (1-2 nm) in size (nanocrystallites) while the Sm layers appear to be amorphous. The diffuse structure of the Sm layers and the interfaces is especially obvious towards the top of the micrograph and away from the substrate.

The Effect of Sm Layer Thickness. Low Angle $\mathrm{X}$-ray Diffraction Profiles. Figure 6 shows a set of low angle $\mathrm{x}$-ray diffraction patterns for multilayer films with a structure of $20[\mathrm{Co}(4.2 \mathrm{~nm}) / \mathrm{Sm}(\mathrm{x} \mathrm{nm})]$ where $x$ ranges from 1.2 to $7.5 \mathrm{~nm}$. Computer simulation of the data for $\mathrm{x}=1.2 \mathrm{~nm}$ is included for comparison. From the figure,

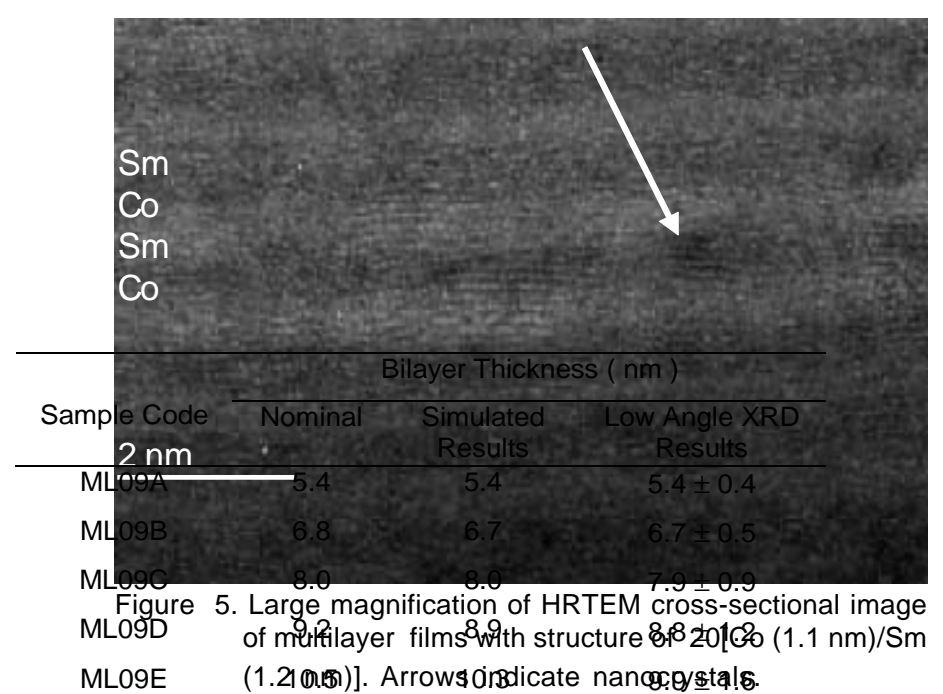

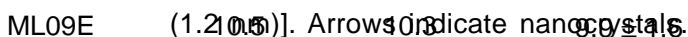

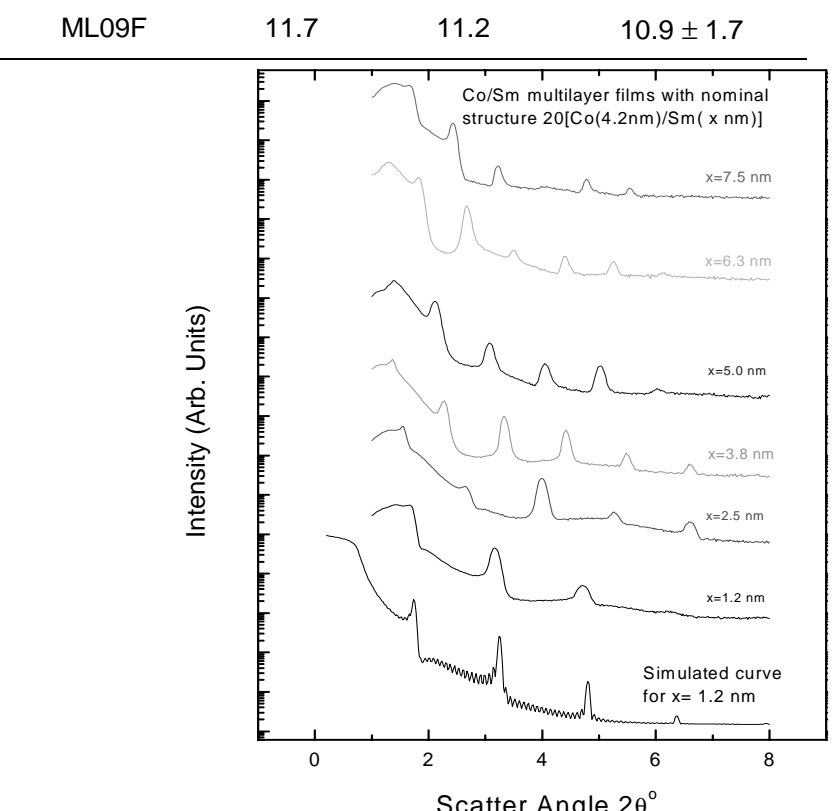

Figure 6. Low angle $x$-ray diffraction profiles of multilayers of $20[\mathrm{Co}(4.2 \mathrm{~nm}) / \mathrm{Sm}(\mathrm{x} \mathrm{nm})]$, where $\mathrm{x}$ ranges from 1.2 to $7.5 \mathrm{~nm}$.
Bragg maxima are clearly observed even at relatively high diffraction angles, suggesting the periodic nature of the samples. For all the multilayer films the interface (Kiessig) fringes from the top and bottom surfaces of the multilayer film stack are not very clear, suggesting that these surfaces are less well-defined. With increasing Sm layer thickness, diffraction peaks shift to a smaller value of angle of incidence suggesting that the bilayer thickness is increased. From the position of low angle XRD peaks shown in Figure 6 , the thickness of the periodic layer (bilayer) for all samples could be calculated. The nominal value of the $\mathrm{Co}$ and Sm layer thicknesses was estimated based on the deposition rates of $\mathrm{Co}$ and $\mathrm{Sm}$.

From the simulated reflectivity pattern analysis, it was found, as an example, that the thickness of a periodic bilayer was $8 \mathrm{~nm}$, which corresponds to the thickness of Co and Sm layers of $4.2 \mathrm{~nm}$ and $3.8 \mathrm{~nm}$ respectively. From low angle XRD measurement, it was found that the thickness of that periodic bilayer was estimated from the position of Bragg peaks as about $7.9 \mathrm{~nm}$. Table 2 shows in comparison the excellent agreement between nominal, simulated and experimental bilayer thickness of the multilayers. However, it can be seen that at higher bilayer thicknesses the experimental and simulated values fall below the nominal value due to interface roughness or mixing.

Magnetic Properties. Figure 7 shows the saturation magnetization values of the 20 [Co $(4.2 \mathrm{~nm}) /$ $\mathrm{Sm}(\mathrm{x} \mathrm{nm})]$ multilayers where $\mathrm{x}$ ranges from 1.2 to 7.5 $\mathrm{nm}$. The theoretical values are included for comparison. Measurements of the saturation magnetization at room temperature show that the magnetization per unit volume of the multilayer films decreases when the Sm layer thickness is increased. This result reflects the fact that higher Sm layer thickness will increase the amount of nonmagnetic element $\mathrm{Sm}$ in the multilayer thus lowering its magnetization. Compared to theoretical values, it is

Table 2. Bilayer thickness of Co/Sm multilayer films with structure of $20[\mathrm{Co}(4.2 \mathrm{~nm}) / \mathrm{Sm}(\mathrm{x} \mathrm{nm})]$. 


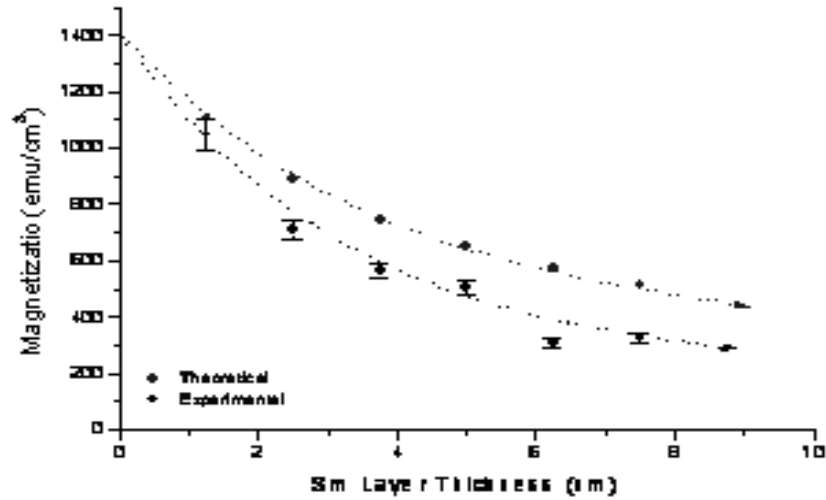

Figure 7. Magnetization of $20[\mathrm{Co}(4.2 \mathrm{~nm}) / \mathrm{Sm}(\mathrm{x} \mathrm{nm})]$ multilayers as a function of $\mathrm{Sm}$ layer thickness; the red line represents theoretical values calculated from equation (2).

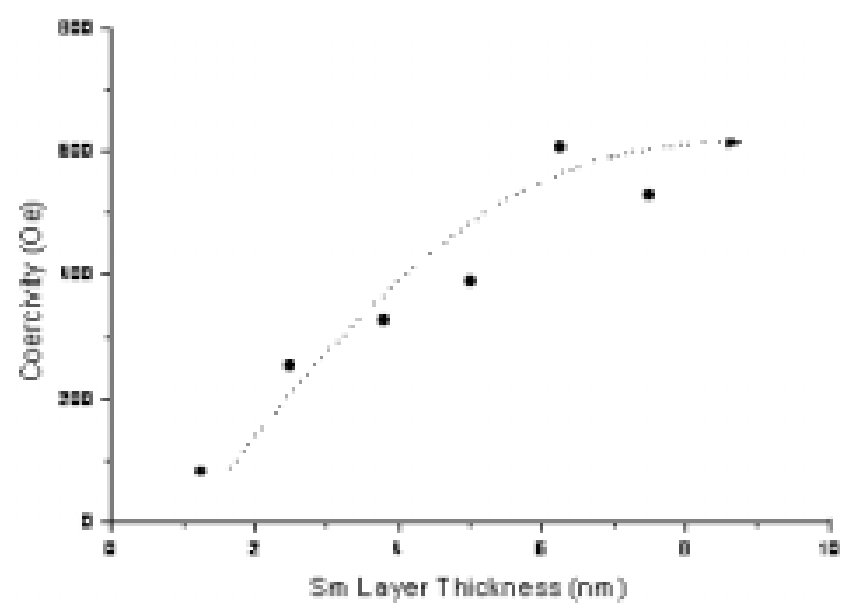

Figure 8. Coercivity of $\mathrm{Co} / \mathrm{Sm}$ multilayer films with nominal structure of $20[\mathrm{Co}(4.2 \mathrm{~nm}) / \mathrm{Sm}(\mathrm{x} \mathrm{nm})]$, where $\mathrm{x}$ ranges from 1.2 to $7.5 \mathrm{~nm}$.

obvious that the measured saturation magnetization exhibits lower values. This result may be ascribed to the intermixing of $\mathrm{Co}$ and $\mathrm{Sm}$ layers in the formation of a thin magnetic or nonmagnetic CoSm 'alloy'.

Figure 8 is a plot of the coercivity of these multilayer films as a function of Sm layer thickness. It can be seen that the coercivities of the multilayer films increases when Sm layer thicknesses increase. The increase in coercivity with increasing samarium layer thickness can be interpreted as being due to surface roughness or mixing, which increases when the Sm layer thickness is increased. This phenomenon leads to the formation a $\mathrm{Co}_{100-x} \mathrm{Sm}_{\mathrm{x}}$ alloy at the interface between $\mathrm{Co}$ and $\mathrm{Sm}$ layers. This effect is supported by the results shown in the low angle XRD measurement shown in Figure 6 in which the diffraction peaks become slightly broader as Sm layer thickness is increased. It also mirrors the coercivity behaviour in Figure 8 where a relative preponderance of cobalt leads to lower coercivities.
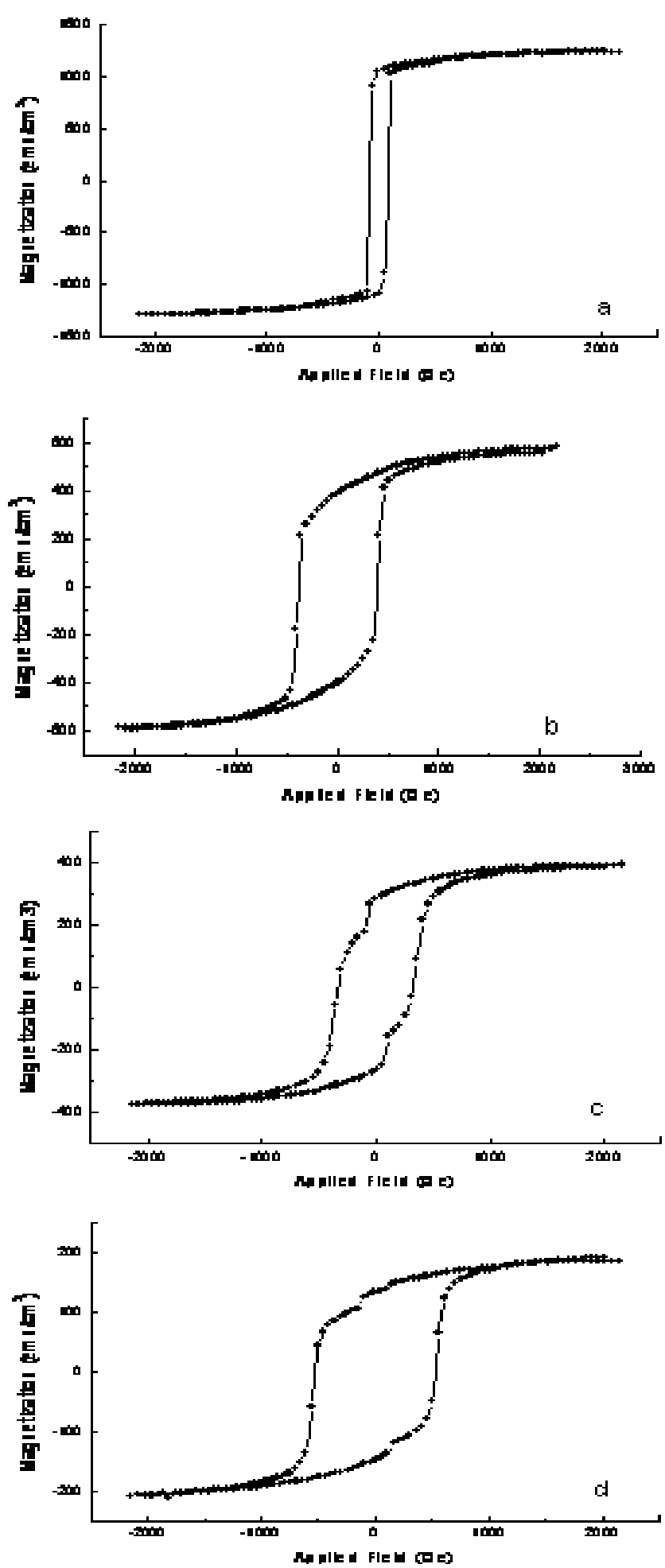

Figure 9. Hysteresis loops at room temperature for multilayer of $20[\mathrm{Co}(4.2 \mathrm{~nm}) / \mathrm{Sm}(\mathrm{x} \mathrm{nm})]$ with (a) $\mathrm{x}=1.2$, (b) $\mathrm{x}=3.8$, (c) $\mathrm{x}=5.0$ and $(\mathrm{d}) \mathrm{x}=7.5$

Figure 9 shows typical hysteresis loops measured at room temperature for as-deposited multilayer films. As the Sm layer thickness is increased the shape of the hysteresis loop changes from high (square-like) to low loop squareness. The hysteresis loop of the multilayer films with Sm layer thickness of $1.2 \mathrm{~nm}$ is nearly square with a coercivity of $84 \mathrm{Oe}$. Above this 
thickness, the hysteresis loops becomes progressively less square. For the multilayer film with $\mathrm{Sm}$ layer thickness of $5.0 \mathrm{~nm}$ the hysteresis loop exhibits a small kink in both field directions, i.e., at near zero field. When the Sm layer thickness is increased further to $7.5 \mathrm{~nm}$, again the hysteresis loop shows a small kink near zero field indicating that the magnetic layers are well separated and non-coherent rotation or the presence of a second phase is certainly established.

Microstructural Properties. Figure 10 shows a cross-sectional image of the multilayer film $20[\mathrm{Co}(4.2 \mathrm{~nm}) / \mathrm{Sm}(3.8 \mathrm{~nm})]$, along with the corresponding diffraction pattern. The periodic $\mathrm{Co}$ and $\mathrm{Sm}$ layers are clearly seen in this image. The apparent bilayer thickness of the Co and Sm estimated from the HRTEM micrograph is less than the nominal value of $4.2 \mathrm{~nm}$ and $3.8 \mathrm{~nm}$ of Co and Sm respectively. This is due to some interdiffusion between $\mathrm{Co}$ and $\mathrm{Sm}$ layers as discussed above. The thicker, darker layer is the Co layer while the thinner, brighter layer is the $\mathrm{Sm}$ layer.

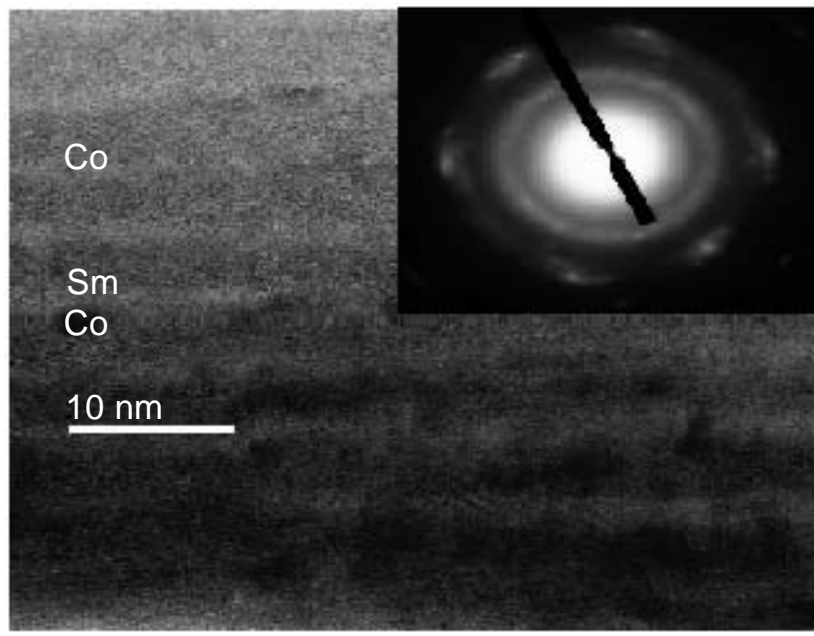

Figure 10. Cross-sectional electron micrograph of a $20[\mathrm{Co}(4.2 \mathrm{~nm})$ /Sm(3.8nm)] multilayer film.

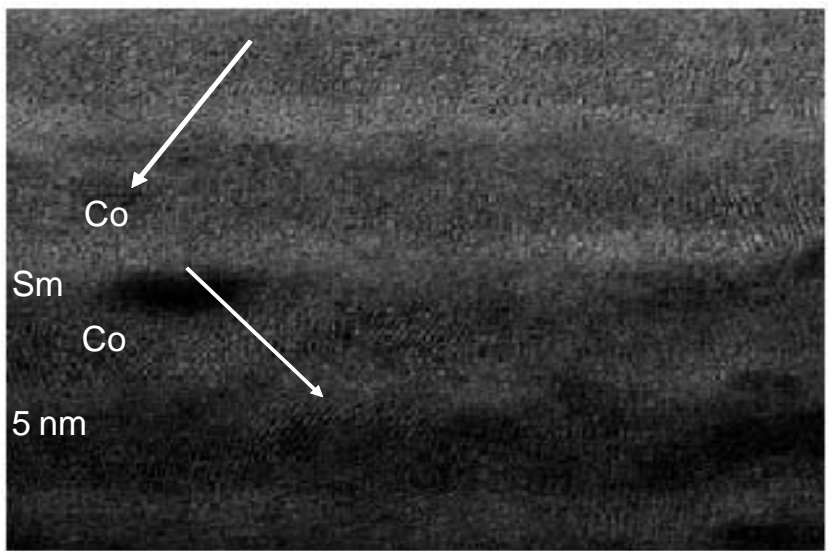

Figure 11. Large magnification HRTEM cross-sectional image of multilayer films with structure of 20 [Co $(4.2 \mathrm{~nm}) / \mathrm{Sm}$ $(3.8 \mathrm{~nm})]$. Arrows indicate nanocrystals.
Another feature that can be noticed from this picture is the long "waviness" of the layers which decreases further away from the substrate, indicating that the flatness of the layers is also increasing but the sharpness of the interfaces becomes less defined with distance from the substrate. The electron diffraction pattern (inserted in Figure 10) from this multilayer film reveals two broad rings associated with an amorphous structure. The first diffuse ring comes from Sm spacings, which can be clearly seen in the electron diffraction pattern of a planar sample (Figure 10). The second broad ring exhibits faint arc shaped reflections indicating some texture in the cobalt component of the multilayer.

Figure 11 shows a highly magnified micrograph of this multilayer film. The brighter $\mathrm{Sm}$ rich layer has a thickness that is smaller than its nominal thickness $(3.8 \mathrm{~nm})$. The Co layers contain $3-6 \mathrm{~nm}$ size nanocrystals with similar lattice fringes as indicated by arrows while the Sm layers appears to be amorphous as can be clearly seen. Obviously, the size of nanocrystallities has increased for thicker Co layers compared to multilayer with lower Co layer thickness, as shown in the HRTEM micrograph Figure 5.

\section{CONCLUSION}

Multilayer films of $\mathrm{Co} / \mathrm{Sm}$ were investigated in order to explore their intrinsic properties and to modify their magnetic properties via interdiffusion between the Co and Sm layers. The study revealed that an increase in the Co layer thickness lead to a large decrease in the coercivity with an accompanying increase in the saturation magnetization. This behaviour was attributed to an increase in the amount of the magnetic Co in the multilayer. The $d M$ studies showed that the exchange interaction among the grains in the layers is stronger for thicker Co layers and results in significant reduction of the coercivity of the multilayers. However, for thinner Co layers the $d M$ shows both broad positive and negative peaks. This could be due to exchange coupling between the Co layers but there is also strong dipolar coupling which moderates the exchange coupling and becomes obvious at large fields. This behaviour was interpreted as being due to the separation of the Co layers that is close enough for significant magnetostatic dipolar coupling. The multilayer structure observed by electron microscopy (HRTEM) revealed a well-defined layer structure. Low angle $X$-ray diffraction profiles supported these results through well-defined Bragg peaks although 
Kiessig fringe are not always very clear. The variation of Sm layer thickness revealed that an increase in the Sm thickness lead to an increase in the coercivity, accompanied by a decrease in the saturation magnetization. The enhancement of coercivity can be interpreted as being due to an increase in the roughness and mixing of the layers.

\section{ACKNOWLEDGMENTS}

The author would like to thank Philip J. Grundy of the University of Salford, Manchester UK for performing the sputtering and experiments.

\section{REFERENCES}

Antel Jr, W.J., Laidler, H. \& Marrows, C.H. 2001. Interaction effects in disordered multilayers. J. Appl. Phys 89: 70717073.

Benaissa, M. \& Krishnan, K.M. 1998. Magnetic anisotropy and its microstructural origin in epitaxially grown SmCo thin films. IEEE Tans. Magn 34: 1204-1206.

Cadieu, F.J., Cheng, T.D., Wickramasekara, L. Kamprath, N., Hedge, H. \& Liu, N.C. 1987. The magnetic properties of high iHc SmCo, NdFeB, and SmTiFe films crystallized from amorphous deposits. J. Appl. Phys 62: 3866-3872.

Charap, S.H., Lu, P.L. \& He, Y. 1997. Thermal stability of recorded information at high densities, IEEE Trans. Magn 33: 978-983.

Cui, F.Z. Fan, Y.D., Wang, Y., Vredenberg, A.M., Draaisma, H.J.D. \& Xu, R. 1990. A new magnetic multilayer system: Iron bismuth. J. Appl. Phys 68: 701-704.

Geiss, V., Kneller, E. \& Nest, A. 1982. Magnetic behavior of amorphous Sm-Co Alloy film. J. Appl. Phys A27: 79-88.
Gronou, M. Goeke, H., Schuffler, D. \& Sprenger, S. 1983. Correlation between domain wall properties and material parameters in amorphous SmCo-films. IEEE Trans, Magn 19: 1653-1655.

Majeticch, S.A., Chowdary, K.M. \& Kirkpatrick, E.M. 1998. Size and interaction effects in the magnetization reversal in SmCo5 nanoparticles. IEEE Trans. Magn 34: 985-988.

Murdock, E.S., Simon, R. \& Davidson, R. 1992. Roadmap for 10Gbit/in2 Media Challenges, Paper JA-01, Intermag '92, St. Louis, Missouri USA.

Petford-Long, K.A., Grundy, P.J. \& Jakubovics, J.P. 1990. Structure and magnetic properties of Co based multilayers. IEEE Trans. Magn 26: 2733:2735.

Robert, P. 1991. 'Thin Film Process II', Edited by J.L. Vossen and W. Kern, Academic Press, Inc, pp 177.

Romero, S.A., Cornejo, D.R., Rhen, F.M., Neiva, A.C., Tabacniks, M.H. \& Missell, F.P. 2000. Magnetic properties and underlayer thickness in $\mathrm{SmCo/Cr}$ films. J. Appl. Phys 87: 6965-6967.

Sigleton, E.W., Shan, Z.S., Jeong, Y.S. \& Sellmyer, D.J. 1995. Magnetic switching volumes of CoSm thin film for high density longitudinal recording. IEEE Trans. Magn 31: 2743-2745.

Stearns, M.B., Lee, C.H. \& Groy, T.L. 1989. Structural studies of Co/Cr multilayered thin film. Phys. Rev B40: 8256-8269. Theuerer, H.C., Nesbitt, E.A. \& Bacon, D.D. 1969. High Coercive Force Rare Earth Alloy Films by Getter Sputtering. J. Appl. Phys 40: 2994-2996.

Velu, E.M.T. \& Lambeth, D.N. 1992. High density recording on $\mathrm{SmCo} / \mathrm{Cr}$ thin film media. IEEE Trans. Magn 28: 3249-3251.

Velu, E.M.T. \& Lambeth, D.N. 1991. CoSm based high coercivity thin films for longitudinal recording. J.Appl. Phys 69: 51755177.

Velu, E.M.T., Lambeth, D.N., Thornton, J.T. \& Russel, P.E. 1994. AFM structure and media noise of CoSm/Cr thin films and hard disk. J. Appl. Phys 75: 6132-6134.

Yan, M.L., Shan, Z.S., Liu, Y. \& Sellmyer, D.J. 1997. High coercivity SmFeAIC thin films fabricated by multilayer sputtering. IEEE Trans. Magn 33: 3706-3708. 